\title{
Figures, Illustrations, and Tables
}

I.1 Frank G. Huntress, San Antonio Express publisher, c. 192920

I.2 William M. McIntosh, San Antonio Light publisher, 192622

1.1 Mexican cotton pickers, Béxar County, August 193033

1.2 Mission San José and the old granary undergoing CWA repairs, $1934 \quad 58$

2.1 Mexican jobless march, downtown San Antonio, $1930 \quad 65$

2.2 Ignacio E. Lozano, La Prensa publisher, film star Antonio Moreno, and Mexican singer Alfonso Ortiz Tirado, 193068

3.1 Matilde Elizondo and Salome Rodriguez, Mexican repatriation, $1931 \quad 112$

3.2 Customers of San Antonio's failed City-Central Bank and Trust Co., $1931 \quad 131$

4.1 Domingo Cortinas, San Antonio Central Relief Committee worker, 1933137

Table 4.1 La Prensa Circulation by Region 144

5.1 Rafael Romero, San Antonio Light newsboy, $1925 \quad 165$

A.1 San Antonio Daily Newspaper Circulation 184

A.2 San Antonio Sunday Newspaper Circulation 185 
THIS PAGE INTENTIONALLY LEFT BLANK 